\title{
Recent ATLAS Results in Charmonium Production
}

\author{
Pavel Řezníček*† \\ IPNP, Faculty of Mathematics and Physics, Charles University, \\ V Holešovičkách 2, 18000 Prague 8, Czech Republic \\ E-mail: reznicek@ipnp.troja.mff.cuni.cz
}

Production of heavy flavoured hadrons can serve as a probe of QCD processes and, in the case of production in heavy ion collisions, also as a probe of the properties of deconfined quark-gluon plasma. This paper describes the latest results in the production of charmonium in the ATLAS experiment at the Large Hadron Collider. The presented analyses include productions of $J / \psi$ and $\psi(2 \mathrm{~S})$ in $p p$ collisions and $\sqrt{s}=7,8$ and $13 \mathrm{TeV}$, and production of $J / \psi, \psi(2 \mathrm{~S})$ and $\Upsilon(\mathrm{nS})$ in $p p$ and $p+\mathrm{Pb}$ collisions at $\sqrt{s}=5.02 \mathrm{TeV}$. In both the analyses prompt and non-prompt productions are disentangled. In the latter analysis production suppression in $p+\mathrm{Pb}$ with respect to $p p$ collisions is measured and emphasis is put on the collision centrality dependence.

The International Conference on B-Physics at Frontier Machines - BEAUTY2018

6-11 May, 2018

La Biodola, Elba Island, Italy

\footnotetext{
* Speaker.

${ }^{\dagger}$ On behalf of the ATLAS collaboration
} 


\section{B-physics in the ATLAS experiment}

The B-physics program in the ATLAS experiment [1] covers a wide range of tests of the Standard Model predictions and of New Physics searches. It consists of precision measurements of $b$ hadron decays, heavy flavour spectroscopy and heavy flavour production. The later in particular tests predictions of the quantum chromodynamics (QCD).

While ATLAS is one of general purpose experiments at the Large Hadron Collider (LHC) [2], the relatively low transverse momentum $p_{\mathrm{T}}$ of the produced $b$-hadrons requires attention to achieve a sufficient suppression of backgrounds. For this reason, the ATLAS B-physics analyses focus on muonic final states, allowing for low- $p_{\mathrm{T}}$ trigger thresholds, and on decays fully reconstructable by the tracking system, providing better purity in the offline analysis.

This paper summarizes the latest results in the production of charmonium.

\section{The ATLAS experiment}

The ATLAS experiment uses a general purpose detector system of almost $4 \pi$ coverage in solid angle. The detector has cylindrical geometry and is forward-backward symmetric. Nearest to the interaction point (IP) is the tracking system, followed by electromagnetic and hadronic calorimeters, while the most distant from the IP is the muon detector system. The tracking system (ID), immersed in $2 \mathrm{~T}$ solenoidal magnetic field and covering pseudorapidity ${ }^{1}|\eta|<2.5$, consists of a silicon pixel detector, silicon strip detector and a transition radiation tracker. The electromagnetic calorimeter is a liquid-argon sampling calorimeter. The same technology is used for the end-cap (large rapidity) hadronic calorimeters, while the central region is covered by a steel-scinillator tile calorimeter. The muon detectors, placed in $0.5 \mathrm{~T}$ toroidal magnetic field and covering $|\eta|<2.7$, are realized by monitored drift tubes and catode strip chambers for precise mesurements, while the fast triggering is served by resistive plate chambers and thin gap chambers detectors.

The ATLAS trigger system reduces data rate from $20 / 40 \mathrm{MHz}$ to recording rate of up to $~$ $1 \mathrm{kHz}$. The system is based on the following stages: fast level-1 (L1) hardware trigger relying on simple signatures, and more sophisticated software trigger (HLT) using partially reconstructed data around the L1 signal. The trigger for the analyses presented in this paper searches for $J / \psi \rightarrow$ $\mu^{+} \mu^{-}$decay signature: in $p p$ collisions requiring two muon with $p_{\mathrm{T}}$ thresholds of $4 \mathrm{GeV}$ at the L1. At the HLT level, the dimuon signature is confirmed by precision detectors and a dimuon vertex fit is performed, applying selection on the vertex quality and keeping only dimuons with the invariant mass close to the $J / \psi$ world average value. In the $p+\mathrm{Pb}$ collisions two muons with $p_{\mathrm{T}}>2 \mathrm{GeV}$ are required, with at least one detected at L1.

\section{3. $J / \psi$ and $\psi(2 S)$ production in $p p$ collisions at 7,8 and $13 \mathrm{TeV}$}

The production of charmonium offers an unique opportunity in trying to understand strong interactions. There are two distinct charmonium production mechanisms at the LHC: prompt, where

\footnotetext{
${ }^{1}$ ATLAS uses a right-handed coordinate system with its origin at the nominal interaction point in the centre of the detector and the $z$-axis along the beam pipe. The $x$-axis points from the IP to the centre of the LHC ring, and the $y$-axis points upward. Cylindrical coordinates $(r, \phi)$ are used in the transverse plane, $\phi$ being the azimuthal angle around the $z$-axis. The pseudorapidity is defined in terms of the polar angle $\theta$ as $\eta=-\ln \tan (\theta / 2)$.
} 
charmonium is produced directly in the $p p$ interactions or through feed-down decays of heavier states, and non-prompt, when charmonium comes from decays of $b$-hadrons. Studying $\psi(2 \mathrm{~S})$ production alongside the $J / \psi$ one allows for disentangling effects from feed-down processes. While around $35 \%$ of prompt $J / \psi$ come from the feed-down decays, the prompt $\psi(2 \mathrm{~S})$ are almost all produced directly.

The analysis [3] is based on data recorded at the LHC during $p p$ collisions at $\sqrt{s}=7 \mathrm{TeV}$ and $8 \mathrm{TeV}$. The data samples correspond to integrated luminosities $\left(L_{\mathrm{int}}\right)$ of $2.1 \mathrm{fb}^{-1}$ and $11.4 \mathrm{fb}^{-1}$. Dimuon events passing trigger conditions mentioned above, are required to contain two oppositecharged muon tracks within $|\eta|<2.3$, that are compatible with the triggered objects by means of $\sqrt{\Delta \eta^{2}+\Delta \phi^{2}}<0.01$. The two muons have to originate in a common vertex of fit quality $\chi^{2}<$ 20. Only dimuons with $p_{\mathrm{T}}(\mu \mu)>8 \mathrm{GeV}$ and rapidity $y(\mu \mu)<2$ are kept.

The differential cross-sections are extracted from signal event yields corrected for the detector geometrical acceptance, trigger efficiency, muon identification and the reconstruction efficiency. In order to disentangle the prompt and non-prompt $J / \psi$ and $\psi(2 \mathrm{~S})$ signal and the prompt and non-prompt backgrounds, an unbinned maximum likelihood fit to the dimuon invariant mass and the pseudo-proper decay time distributions is performed. The pseudo-proper decay time is constructed as $\tau=L_{x y} m(\mu \mu) / p_{\mathrm{T}}(\mu \mu)$, where $L_{x y}$ is the signed projection of the distance of the dimuon decay vertex from the primary vertex onto its transverse momentum. An example of such a fit is demonstrated in two projections in figure 1. Since the spin alignment of the charmonium production in the ATLAS experiment has not been measured yet, the results in the plots of the paper are defined for the unpolarized case. However, correction factors for six other spin alignment scenarios are provided.
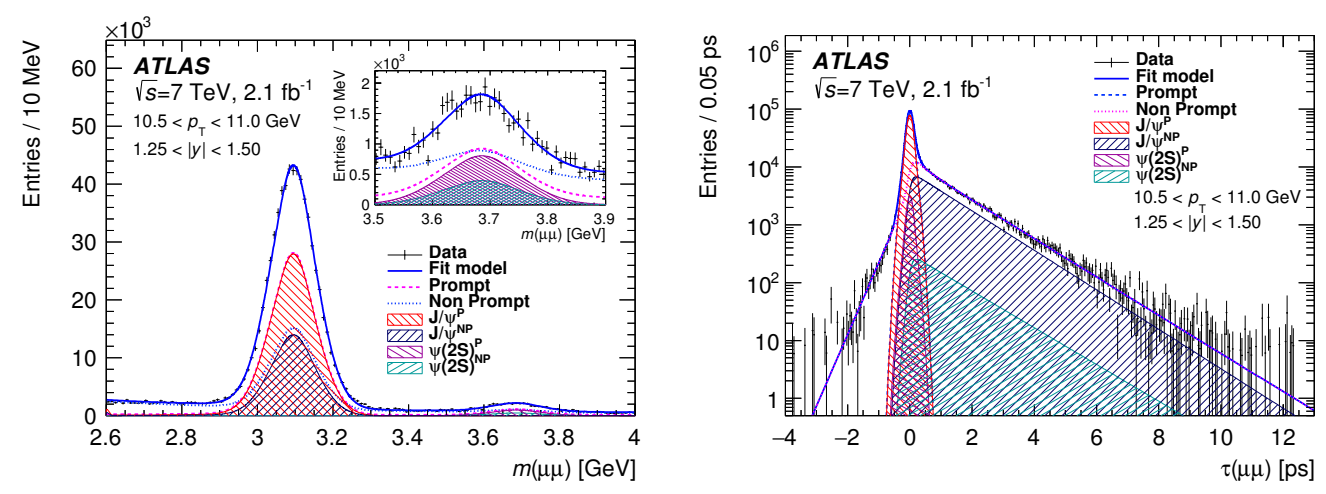

Figure 1: Projections of the fit result over the mass (left) and pseudo-proper decay time (right) distributions for data collected at $7 \mathrm{TeV}$ for one typical interval. The projections of the individual components of the fit result are shown alongside the data. Figures taken from [3].

The analysis presents differential cross-section of the prompt and non-prompt $J / \psi$ and $\psi(2 \mathrm{~S})$ production, as a function of the dimuon $p_{\mathrm{T}}$ and rapidity. Similar kinematic dependencies of the nonprompt production fraction are studied. The prompt $J / \psi$ cross-section is in good agreement with non-relativistic QCD (NRQCD) predictions across the whole $p_{\mathrm{T}}$ range, without rapidity dependence. The prompt $\psi(2 \mathrm{~S})$ production also well agrees with the NRQCD predictions, but with slight theory overestimation at high $p_{\mathrm{T}}$ (see figure 2 top). In the case of non-prompt produc- 

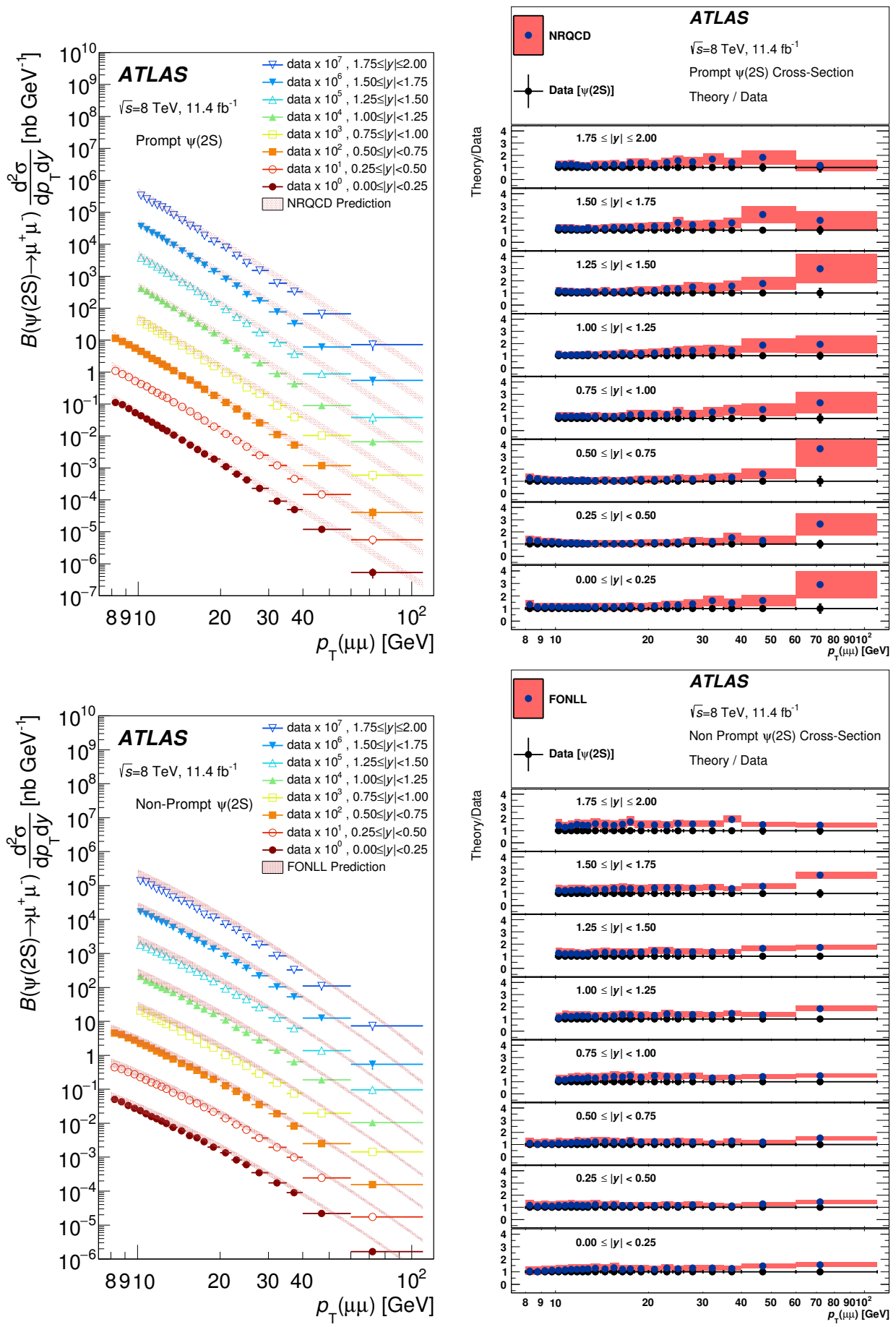

Figure 2: The differential prompt cross-section times dimuon branching fraction of prompt (top) and nonprompt (bottom) $\psi(2 \mathrm{~S})$ as a function of $p_{\mathrm{T}}(\mu \mu)$ for each slice of rapidity, taken from [3]. The $8 \mathrm{TeV}$ results are shown. The centre of each bin on the horizontal axis represents the mean of the weighted $p_{\mathrm{T}}$ distribution. The horizontal error bars represent the range of $p_{\mathrm{T}}$ for the bin, and the vertical error bar covers the statistical and systematic uncertainty. Theory predictions are also shown. The figures on the left present the ratio of the theory predictions to data. 
tion, fixed order next-to-leading logarithm (FONLL) calculations predict slightly harder $p_{\mathrm{T}}$ spectra for both $J / \psi$ and $\psi(2 S)$ (see figure 2 bottom). The ratio of the prompt $\psi(2 \mathrm{~S})$ to the prompt $J / \psi$ is found flat across the whole $p_{\mathrm{T}}$ range. The results are consistent with the CMS [4] and LHCb [5] measurements in the overlapping kinematic regions.

The prompt $J / \psi$ fraction dominates at low- $p_{\mathrm{T}}$, but non-promptly produced $J / \psi$ exceed prompt at around $p_{\mathrm{T}}$ of $20 \mathrm{GeV}$, as is shown at figure 3 (left). The non-prompt fraction has also been extracted from the newer $\sqrt{s}=13 \mathrm{TeV}$ data [6], as demonstrated in figure 3 (top right). The comparison of this fraction measured at different $\sqrt{s}$ collision energies shows increasing tendency (see figure 3 bottom right).
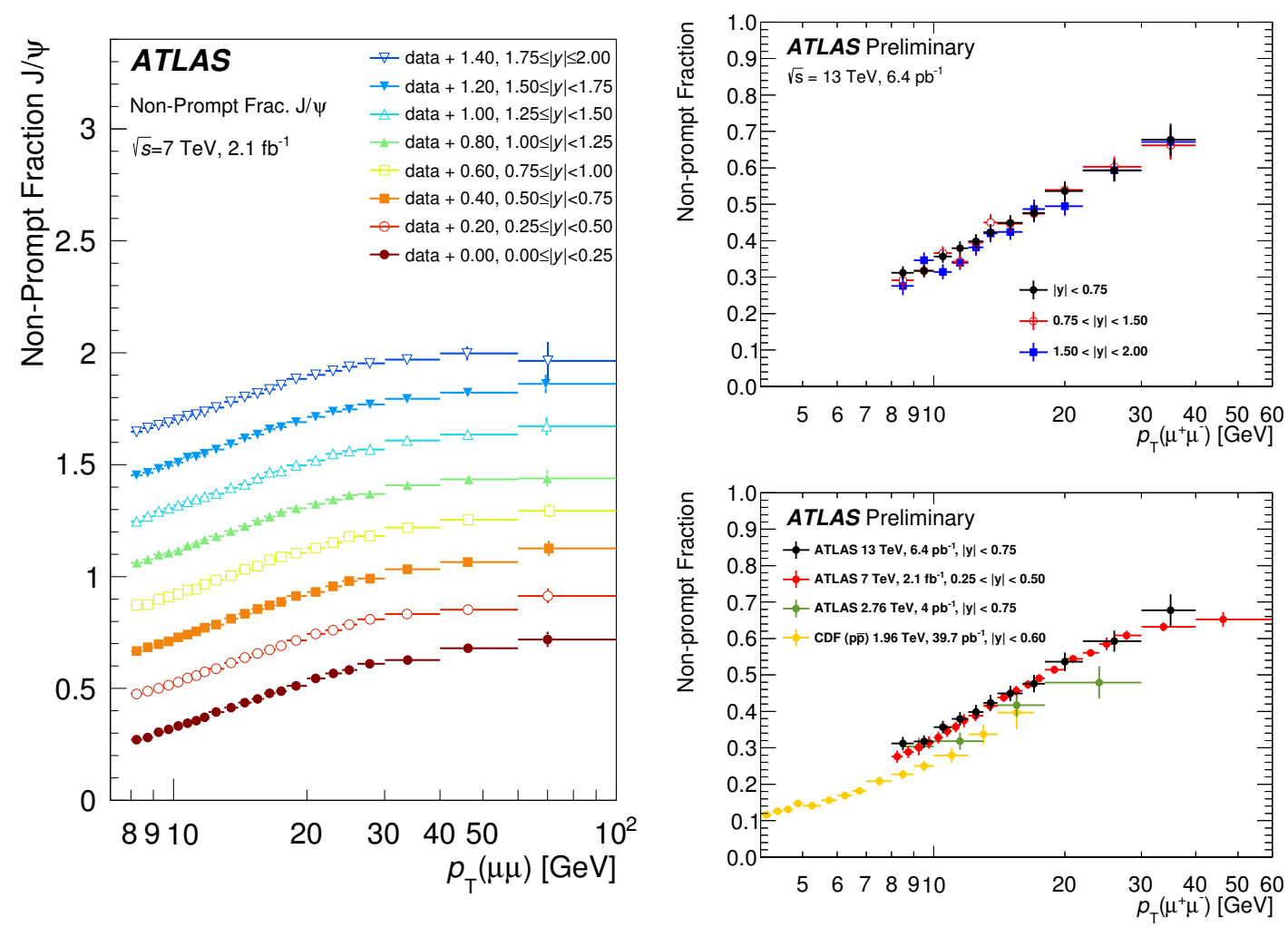

Figure 3: The non-prompt fraction of $J / \psi$ as a function of $p_{\mathrm{T}}(\mu \mu)$ for each slice of rapidity are shown for the $7 \mathrm{TeV}$ (left) [3] and $13 \mathrm{TeV}$ [6] results (top right). The right bottom figure presents the comparison of the non-prompt fraction of $J / \psi$ with the previous measurements from ATLAS [7] and from CDF [8].

\section{4. $J / \psi, \psi(2 S)$ and $\Upsilon(\mathrm{nS})$ production in $p p$ and $p+\mathrm{Pb}$ collisions at $5.02 \mathrm{TeV}$}

The measurement of quarkonuim production in heavy ion collisions is a valuable probe of the deconfined quark-gluon plasma. A possible suppression of the production (melting) can provide information about the temperature and degree of the deconfinement. However, enhancement could also appear, e.g. when quarkonium is formed due to recombination of $c \bar{c}$ quarks from the medium. Studies of both prompt and non-prompt quarkonium are important as the nonprompt allows to study $b$ quark propagation through the medium. The $b$ quarks rather suffer from 
different suppression mechanism (energy loss by collisional or radiative processes), while $c \bar{c}$ can be inhibited by color screening from the medium. Study of production within $p+\mathrm{Pb}$ collisions, where a formation of a large region of hot and deconfined matter is not expected, allows to probe Cold Nuclear Matter (CNM) effects. The CNM quarkonium suppression effects can either be at the initial state (modifications of the nuclear parton distribution functions (nPDF), parton saturation in the incident nucleus, or parton energy loss through interactions with the nuclear medium), or final state (the absorption of the heavy $q \bar{q}$ pairs).

The presented analysis [9] uses data recorded at the LHC during $p p$ and $p+\mathrm{Pb}$ collisions at $\sqrt{s}=5.02 \mathrm{TeV}$. The data samples correspond to $L_{\mathrm{int}}$ of $25 \mathrm{pb}^{-1}$ and $28 \mathrm{nb}^{-1}$. Triggered dimuon events (see above) are required contain two opposite-charged muon tracks within $|\eta|<2.4$ and satisfying tight muon identification criteria.

A similar method of mass and pseudo-proper decay time fit is used to extract the prompt and non-prompt charmonium. Bottomonium yields are taken from dimuon mass fits only. The quarkonium are assumed to be unpolarized. An important aspect of the analysis is the dependence of the production on event centrality ${ }^{2}$, that characterizes the $p+\mathrm{Pb}$ collision geometry (more or fewer participating nucleons) and is based on the total transverse momentum measured in the forward calorimeter (FCal) on the side of the outgoing $\mathrm{Pb}$ ion beam.

The results include $J / \psi, \psi(2 \mathrm{~S})$ and $\Upsilon(\mathrm{nS})$ production cross-section, relative production of the quarkonium in the $p+\mathrm{Pb}$ to $p p$ collisions (nuclear modification $R$-factor), as well as double-ratio $\rho$ of production of higher quarkonium states to the base (1S) state. The $R$-factor is defined as $R_{p \mathrm{~Pb}}=1 / 208 \cdot \sigma_{p+\mathrm{Pb}}^{O(n \mathrm{~S})} / \sigma_{p p}^{O(n \mathrm{~S})}$ and the $\rho_{p \mathrm{~Pb}}^{O(n \mathrm{~S}) / O(1 \mathrm{~S})}=R_{p \mathrm{~Pb}}(O(n \mathrm{~S})) / R_{p \mathrm{~Pb}}(O(1 \mathrm{~S}))$. All these are studied as a function of the dimuon $p_{\mathrm{T}}$, proton-nucleon centre-of-mass rapidity ${ }^{3}, y^{*}$, and the event centrality. The prompt charmonium production is compatible with the NLO NRQCD prediction, while $\Upsilon(1 \mathrm{~S})$ production is overestimated in $p_{\mathrm{T}}$ below $15 \mathrm{GeV}$. Non-prompt charmonium production agrees with FONLL calculations. The $R$-factors of both the prompt and non-prompt (from $b$-decays) charmonium are consistent with unity, suggesting weak modifications due to CNM effects. However, the $R$-factors of $\Upsilon(1 \mathrm{~S})$ are below unity for $p_{\mathrm{T}}<15 \mathrm{GeV}$, as illustrated on figure 4 (left). No $y^{*}$ dependence is observed in this case (figure 4 right). The observed suppression can be possibly used to study modification of the nPDF relative to of nucleon.

The double-production ratios show a clear suppression of the higher states relative to the promptly produced charmonia and bottomonia. The prompt $\psi(2 \mathrm{~S})$ relative suppression is observed with the significance of $1 \sigma$ and increasing with rapidity (see figure 5 top left). The $\Upsilon(2 S)$ and $\Upsilon(3 S)$ relative suppression is at the level of $2 \sigma$, in $p_{\mathrm{T}}<40 \mathrm{GeV}$ and rapidity range of $-2<y^{*}<1.5$. For all cases the effect is stronger in more central collisions at $1 \sigma$ level, as is shown on bottom figures 5. For the ground state, the production as a function of centrality follows $Z$-boson production. The results thus suggest stronger CNM effects in the excited quarkonium then in the ground states.

The paper also studied trend in self-normalized yields of the ground states as a fucntion of selfnormalized event activity in the FCal, finding a linear correlation with a deviation of $2 \sigma$ at the highest event activity. This result is compatible with CMS observation [10].

\footnotetext{
${ }^{2}$ The smaller centrality values [\%] correspond to more central collisions.

${ }^{3}$ In the $p+\mathrm{Pb}$ collisions the proton-nucleon centre-of-mass rapidity $y^{*}$ was shifted by $\Delta y=0.465$ w.r.t. rapidity in the laboratory frame. The proton and $\mathrm{Pb}$ beam directions were reversed after $60 \%$ of the data taking, keeping convention that proton beam travels in direction of positive $y^{*}$. Data from both data taking periods are used.
} 

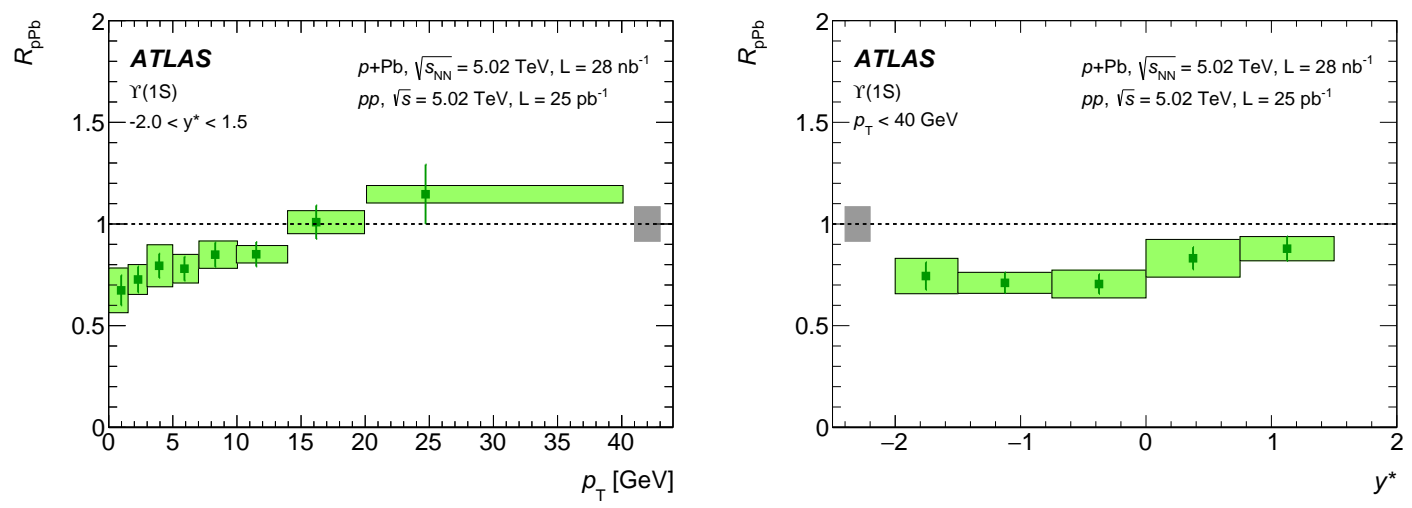

Figure 4: The nuclear modification factor as a function of transverse momentum $p_{\mathrm{T}}$ (left) and centre-of-mass rapidity $y^{*}$ (right) for $\Upsilon(1 \mathrm{~S})$, taken from [9]. The horizontal position of each data point indicates the mean of the weighted $p_{\mathrm{T}}$ or $y^{*}$ distribution. Statistical and uncorrelated and correlated systematic uncertainties are shown with the vertical error bars and colored and gray boxes.
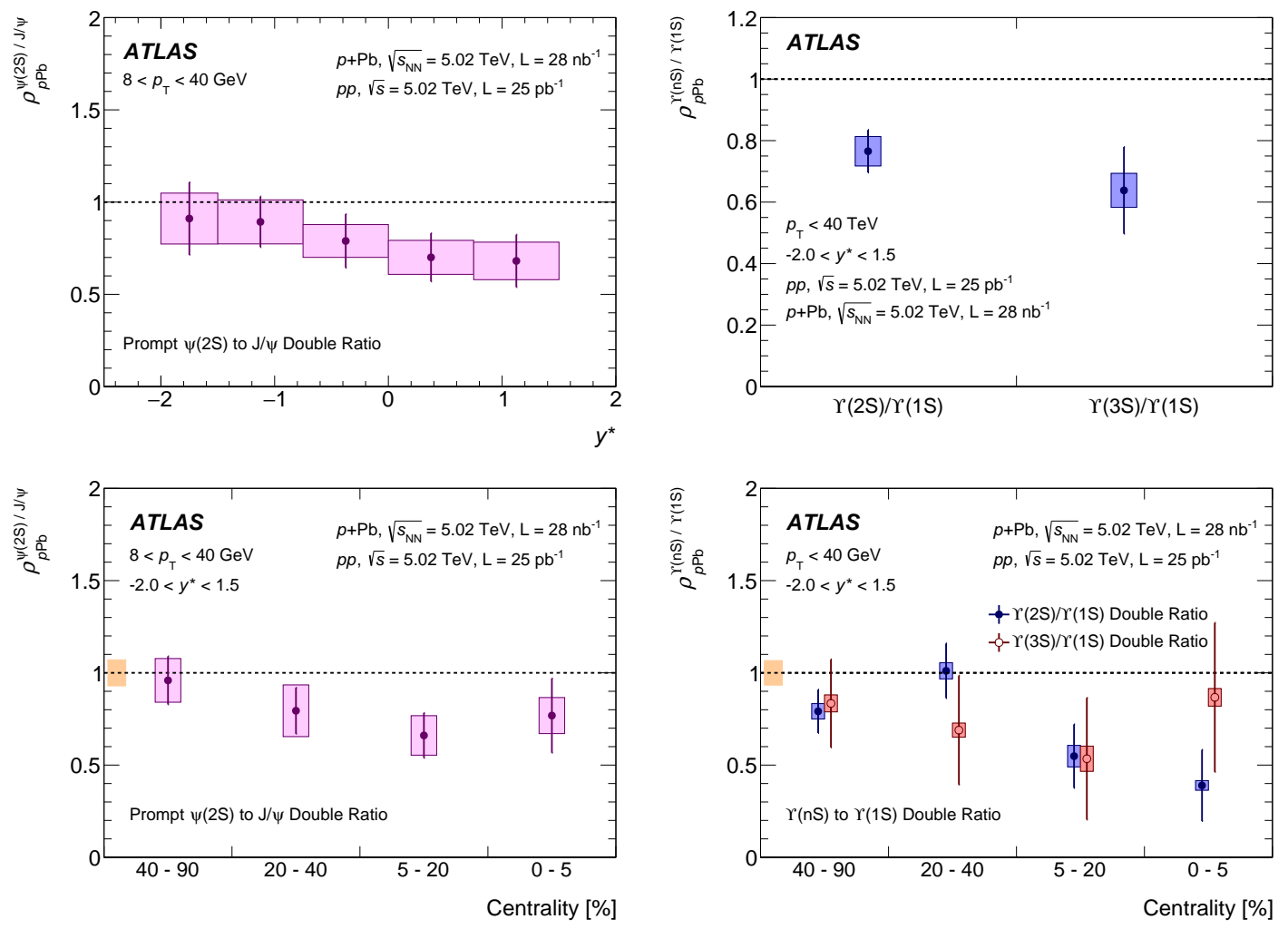

Figure 5: The prompt charmonium production double ratio as a function of the centre-of-mass rapidity $y^{*}$ (top left) and event centrality in $p+\mathrm{Pb}$ collisions (bottom left). The bottomonium double ratios for the whole rapidy range and as a function of the event centrality are shown on the top and bottom right figures. Statistical and uncorrelated systematic uncertainties are shown with the vertical error bars and colored boxes. The leftmost yellow boxes in the bottom figures represent the total uncertainty of the $p p$ reference. Figures taken from [9]. 


\section{Summary}

The recent results of the ATLAS experiment at the LHC in charmonium production are presented. The analysis of $J / \psi$ and $\psi(2 \mathrm{~S})$ in $p p$ collisions and $\sqrt{s}=7$ and $8 \mathrm{TeV}$ includes $p_{\mathrm{T}}$ and rapidity dependence of the differential production cross-section, both for promptly and non-promptly produced charmonia. A good agreement with the QCD predictions is found, except for slight deterioration at high $p_{\mathrm{T}}$. ATLAS recently measured the non-prompt fraction of a charmonium production at $13 \mathrm{TeV}$ in $p p$ collisions. Quarkonium production is also studied in the $p+\mathrm{Pb}$ collisions at $\sqrt{s}=5.02 \mathrm{TeV}$, focusing on the production relative to $p p$ collisions in order to study cold nuclear matter effects. The analysis reveals a suppression of both the prompt excited charmonium and the bottomonium productions, relative to their ground states, which is getting stronger in central collisions. A suppression of $\Upsilon(1 \mathrm{~S})$ production is observed at low- $p_{\mathrm{T}}$ values. On the other side, prompt $J / \psi$ and non-prompt charmonium states are produced at similar rates as in the $p p$ collisions. The signal yields are also studied relative to that of $Z$-boson.

\section{Acknowledgement}

Support for this work has been received from the grant LTT 17018 of the Ministry of Education, Youth and Sports of the Czech Republic, and from the project UNCE/SCI/013 of the Charles University Research Center.

\section{References}

[1] ATLAS Collaboration, The ATLAS Experiment at the CERN Large Hadron Collider, JINST 3 (2008) S08003.

[2] L. Evans and P. Bryant, LHC Machine, JINST 3, S08001 (2008).

[3] ATLAS Collaboration Measurement of the differential cross-sections of prompt and non-prompt production of $J / \psi$ and $\psi(2 S)$ in pp collisions at $\sqrt{s}=7$ and $8 \mathrm{TeV}$ with the ATLAS detector, Eur. Phys. J. C 76 (2016) no.5, 283, arXiv:1512.03657 [hep-ex].

[4] CMS Collaboration, Measurement of $J / \psi$ and $\psi(2 S)$ Prompt Double-Differential Cross Sections in pp Collisions at $\sqrt{s}=7$ TeV, Phys. Rev. Lett. 114 (2015) no.19, 191802, arXiv:1502.04155 [hep-ex].

[5] LHCb Collaboration, Production of $J / \psi$ and $\Upsilon$ mesons in pp collisions at $\sqrt{s}=8 \mathrm{TeV}$, JHEP 1306 (2013) 064, arXiv:1304.6977 [hep-ex].

[6] ATLAS Collaboration, Measurement of the differential non-prompt $J / \psi$ production fraction in $\sqrt{s}=13 \mathrm{TeV}$ pp collisions at the ATLAS experiment, ATLAS-CONF-2015-030.

[7] ATLAS Collaboration, Study of $J / \psi$ and $\psi(2 S)$ production in $\sqrt{s_{N N}}=5.02 \mathrm{TeV} p+P b$ and $\sqrt{s}=2.76 \mathrm{TeV}$ pp collisions with the ATLAS detector, ATLAS-CONF-2015-023.

[8] CDF Collaboration, Measurement of the $J / \psi$ meson and $b$-hadron production cross sections in $p \bar{p}$ collisions at $\sqrt{s}=1960$ GeV, Phys. Rev. D 71 (2005) 032001, arXiv [hep-ex/0412071].

[9] ATLAS Collaboration, Measurement of quarkonium production in proton-lead and proton-proton collisions at $5.02 \mathrm{TeV}$ with the ATLAS detector, Eur. Phys. J. C 78 (2018) no.3, 171, arXiv:1709.03089 [nucl-ex].

[10] CMS Collaboration, Event activity dependence of $Y(n S)$ production in $\sqrt{s_{N N}}=5.02 \mathrm{TeV} p+P b$ and $\sqrt{s}=2.76 \mathrm{TeV}$ p p collisions, JHEP 1404 (2014) 103, arXiv:1312.6300 [nucl-ex]. 